Dominik Exel* und Bernhard Zagar

\title{
Systemdesign und Fehlerabschätzung der radio-akustischen Temperaturmessung
}

\author{
System's design and error limits of radio-acoustic temperature measurement
}

https://doi.org/10.1515/teme-2020-0102

Eingang 31. Dezember 2020; angenommen 24. Januar 2021

Zusammenfassung: Berührungslose Temperatur- und Strömungsgeschwindigkeitsmessung ist von enormer Wichtigkeit, sowohl in der Klimaforschung aber auch bei industriellen Anwendungen. Dabei ist es für die Optimierung der Systeme entscheidend, die zu erwartenden Messfehler abschätzen zu können. In diesem Beitrag wird eine dieser Methoden basierend auf dem radio-akustischen Prinzip (RASS) umfassend untersucht. Messsysteme nach dieser Methode nützen die akustisch-elektromagnetische Wechselwirkung um aus der lokal ermittelten Schallgeschwindigkeit auf die vorherrschende Gastemperatur, aber gegebenenfalls auch auf eine lokale wirkende Komponente der Strömungsgeschwindigkeit in Messrichtung $\mathrm{zu}$ schließen. Um das Ziel zu erreichen, das Messsystem in seiner Gesamtheit zu analysieren, wird zunächst die sogenannte RASS-Gleichung sowie die notwendige Kollokation der Quellen diskutiert. Anhand des Ausdrucks der Empfangsleistung wird gezeigt, welche Faktoren maßgeblich das Systemverhalten beeinflussen. Eine entsprechende Abstimmung der Schall- bzw. Radar-Wellenlängen und eine perfekte Kollokation deren Quellen sind essentiell für eine ausreichend genaue Schätzung der von der Temperatur abhängigen Doppler-Frequenz. Anschließend wird, nach Wissen der Autoren erstmalig anhand der CramérRao Schranke ein optimales Systemdesign abgeleitet. Dabei zeigt sich, dass sich die Varianz der Temperaturschätzung verkehrt proportional zur 4. Potenz der Anzahl der wechselwirkenden Schallzyklen verhält. Weiters wird demonstriert, dass die Temperatur und die Strömungsgeschwindigkeit nicht unabhängig voneinander geschätzt werden können. Es wird in diesem Beitrag ein Aufbau präsentiert, der es erlaubt die Temperaturen mit einer Standardabweichung von etwa $0,2^{\circ} \mathrm{C}$ zu messen. Zur Demonstration wird eine Temperaturprofilmessung vorgestellt.

*Korrespondenzautor: Dominik Exel, Johannes Kepler Universität Linz, Institut für Elektrische Messtechnik, Altenbergerstraße 69, 4040 Linz, Austria, E-Mail: dominik.exel@jku.at

Bernhard Zagar, Johannes Kepler Universität Linz, Institut für Elektrische Messtechnik, Altenbergerstraße 69, 4040 Linz, Austria
Schlagwörter: Temperaturmessung, RASS, Cramér-Rao Schranke, Doppler-Radar.

Abstract: Contactless temperature and flow velocity measurement is of enormous importance, both in climate research and in industrial applications. It is crucial for the optimization of the systems to be able to estimate the expected measurement errors. In this article, one of the methods the radio-acoustic sounding system (RASS) is extensively investigated. Measurement systems operating on this method use the acoustic-electromagnetic interaction to infer the prevailing gas temperature from the spatially resolved speed of sound, but also, if necessary, to infer the spatially acting component of the flow velocity in the measurement direction. In order to achieve the goal of analyzing the measurement system in its entirety, the socalled RASS equation and the necessary collocation condition of the sources are discussed first. The expression of the backscattered power indicates which factors have a decisive influence on the system behavior. Appropriate matching of the sound and Radar wavelengths and perfect collocation of their sources are essential for a sufficiently accurate estimate of the temperature-dependent Doppler frequency. Afterwards, to the best knowledge of the authors, an optimal system design is derived for the first time based on the Cramér-Rao lower bound. It can be seen that the variance of the temperature estimate is inversely proportional to the 4 th power of the number of interacting sound cycles. It is also demonstrated that the temperature and the flow velocity cannot be estimated independently of one another. In this article a setup is presented that allows the temperatures to be measured with a standard deviation of about $\pm 0,2^{\circ} \mathrm{C}$. A temperature profile measurement is presented as a demonstration.

Keywords: Temperature estimation, RASS, Cramér-Rao lower bound, Doppler radar.

\section{Einleitung}

Die Möglichkeit eine Gastemperatur nicht nur berührungslos sondern auch über eine nennenswerte Distanz mes- 
sen zu können erlaubt gerade in rauen industriellen Umgebungen Einsatzbereiche, die alternativ nur sehr schwer abzudecken wären. So bietet die Möglichkeit in der Gicht eines Hochofens ein vollständiges Temperarturprofil zu bestimmen, Einflussmöglichkeiten auf die Qualität von Roheisen. Aber auch die kontinuierliche Überwachung der Temperatur entlang eines Hubbalken- oder Durchlaufofens erlaubt die Realisierung entscheidender Qualitätsvorteile. Diese Methode zur berührungslosen Temperaturmessung erlaubt, im Gegensatz zur häufig verwendeten und auf die Messung der Oberflächentemperatur von Körpern beschränkten Pyrometrie den Einsatz in Gasen. Ebenso wie für die Pyrometrie ist auch hier im weitesten Sinne ein 'Sichtzugang' notwendig. Auch die Möglichkeit zur Messung an bewegten, schwer zugänglichen Objekten stellen klare Vorteile dar.

Bei der klassischen akustischen Gasthermometrie wird die Temperatur-Schallgeschwindigkeitsbeziehung, welche für ein bekanntes Gasgemisch einen eindeutigen Zusammenhang aufweist, ausgenützt. Wird in Luft bei annähernd Standardbedingungen, also in einem von einem idealen Gas nur gering abweichenden Medium, gemessen, so lautet der Zusammenhang näherungsweise

$$
c_{\mathrm{a}}=\sqrt{\frac{\kappa \cdot R \cdot \vartheta}{M}}=K_{\mathrm{a}} \cdot \sqrt{\vartheta}
$$

mit $c_{\mathrm{a}}$ der Schallgeschwindigkeit (der Phasengeschwindigkeit) in $\mathrm{m} / \mathrm{s}, \kappa$ dem Isentropenexponent $(\approx 1,40$ für Luft), $R=8,314 \ldots$ der allgemeinen Gaskonstante in $\mathrm{J} /(\mathrm{mol} \mathrm{K})$ und $\vartheta$ der Temperatur in K [4]. Die Konstante $K_{\mathrm{a}}=20,045$ in $\mathrm{m} /(\mathrm{s} \sqrt{\mathrm{K}})$ gilt für das Medium Luft.

Die Schallgeschwindigkeit wird über die Laufzeit $T$ eines Schallpulses, der mehrere Perioden umfasst und damit örtlich mittelt, entlang des Pfades $s$ zwischen den Punkten $A$ und $B$ gemessen. Damit lässt sich rechnerisch eine mittlere Temperatur $\bar{\vartheta}$ entlang des betrachteten Teils $\overline{A B}$ des Signalpfads bestimmen. Aus der Laufzeit $T$, die sich einerseits aus der durchlaufenen Strecke $\overline{A B}$ dividiert durch die gemittelte Geschwindigkeit $\overline{c_{\mathrm{a}}}=\overline{K_{\mathrm{a}} \cdot \sqrt{\vartheta}}$ und andererseits aus dem Integral der mit variabler Geschwindigkeit $c_{\mathrm{a}}(s(\vartheta))$ durchlaufener Streckenabschnitte $d s$ berechnet, folgt:

$$
\begin{gathered}
T=\frac{1}{\overline{K_{a} \cdot \sqrt{\vartheta}}} \cdot \underbrace{\int_{A}^{B} d s}_{\overline{A B}}=\int_{A}^{B} \frac{1}{K_{a} \cdot \sqrt{\vartheta(s)}} d s, \\
\overline{\sqrt{\vartheta}}=\frac{1}{\overline{A B}} \cdot\left\{\int_{A}^{B} \frac{d s}{\sqrt{\vartheta(s)}}\right\}^{-1} .
\end{gathered}
$$

Die Tatsache, dass dadurch nur eine mittlere Temperatur $\overline{\sqrt{\vartheta}}$ ermittelt werden kann, stellt einen Nachteil der akustischen Gastemperaturmessung dar. Durch mehrere Messpfade die sich überkreuzen lassen sich durch tomographische Rekonstruktion Temperaturverteilungen, z. B. im Volumen eines Ofens, darstellen [5, 6, 7]. Im Gegensatz dazu kann mit der RASS Methode mit nur einer Messstelle berührungslos und grob örtlich aufgelöst die Gastemperatur gemessen werden $[8,9]$.

Um diese Messmethode zu analysieren, wird dieser Artikel folgendermaßen gegliedert:

In Abschnitt 2 wird die Funktionsweise des RASS beschrieben und die sogenannte RASS-Gleichung angegeben. Das Blockschaltbild eines Doppler-Radars als eines der Hauptkomponenten, sowie die Signalverarbeitung werden in Abschnitt 3 präsentiert. Weiters wird auch ein geeignetes Signalmodell vorgestellt und das sich ergebende Frequenzschätzproblem diskutiert. In Abschnitt 4 wird die Cramér-Rao Schranke berechnet, aus der sich eine optimale Systemauslegung ableiten lässt. Da von den Autoren bereits in [3] und in [9] Labormessungen bzw. Messungen bei einer nicht perfekten Kollokation der Quellen vorgestellt wurden, werden in diesem Beitrag in Abschnitt 5 neben der Beschreibung des Messablaufes auch neuere Ergebnisse zur Messung eines Temperaturprofiles präsentiert.

\section{Funktionsweise}

Das wesentliche Merkmal eines RASS ist die Kombination einer elektromagnetischen Sende- und Empfangseinheit, mit einem akustischem Sender [10]. Konkret wird eine leistungsstarke, sphärische Ultraschallwelle ausgesandt und mittels eines Doppler-Radars die (radiale) Ausbreitungsgeschwindigkeit dieser, den lokalen Brechungsindex modulierenden, Welle gemessen, siehe Abbildung 1. Propagierender Schall in einem Gas bewirkt lokal eine Expansion bzw. Verdichtung des Ausbreitungsmediums (um $\pm \Delta p(t))$, so dass sich ein Druck von $p(t)=p_{0} \pm \Delta p$ einstellt. Diese Druckschwankung um den statischen Druck $p_{0}$ führt, entsprechend der Gladstone-Dale Beziehung für Gase

$$
\begin{aligned}
\frac{n_{\mathrm{a}}-1}{n_{\mathrm{a}, 0}-1} & =\frac{\rho_{\mathrm{a}}}{\rho_{\mathrm{a}, 0}}=\left(\frac{p_{0} \pm \Delta p}{p_{0}}\right)^{1 / \kappa}, \\
\Delta n_{a} & =\frac{n_{\mathrm{a}, 0}-1}{\kappa \cdot p_{0}} \cdot \Delta p,
\end{aligned}
$$

zu einer lokal wirkenden, sehr geringen Abweichung vom mittleren Brechungsindex $n_{\mathrm{a}}(s)=\overline{n_{\mathrm{a}}} \pm \Delta n_{\mathrm{a}}(s)=1,000292 \pm$ 
$\Delta n_{\mathrm{a}}(\mathrm{s})$. Die Größenordnung kann abgeschätzt werden $(\kappa=$ $1,40, p_{0}=101325 \mathrm{~Pa}, \Delta p=200 \mathrm{~Pa} @ 140 \mathrm{~dB}$ Schalldruckpegel, $\rho_{\mathrm{a}}$ Dichte der Luft) mit $\Delta n_{a}=412 \cdot 10^{-9}$.

Für eine mit dem gleichen Wellenvektor propagierende elektromagnetische Welle stellt der Gradient der Dichte und somit der Gradient des Brechungsindex $\nabla n_{\mathrm{a}}(s)$ einen deterministischen Streuer dar, der mit einer gewissen, relativ geringen Effektivität rückstreuend wirkt [11, 12]. Die propagierende Schallwelle stellt bei geeigneter geometrischer Anordnung also einen bewegten Rückwärtsstreuer für die elektromagnetischen Wellen dar. Da sich die Fortbewegungsgeschwindigkeit der Schallwelle aus der Phasengeschwindigkeit $c_{\mathrm{a}}(s)$ und einer Strömungsgeschwindigkeit $v_{\mathrm{w}}\left(v_{\mathrm{w}}\right.$ ist die Komponente des Geschwindigkeitsvektors $\vec{v}_{\mathrm{w}}$ projiziert in die Ausbreitungsrichtung der elektromagnetischen Welle [14]) zusammensetzt, resultiert daraus eine entsprechende Dopplerfrequenzverschiebung $f_{\mathrm{d}}$, die aus dem Radar-Empfangssignal messtechnisch ausgewertet werden kann $[1,13]$

$$
f_{\mathrm{d}}=\frac{2 \cdot\left(c_{\mathrm{a}}(s)+v_{\mathrm{W}}\right)}{c} f_{\mathrm{e}} .
$$

$f_{\mathrm{e}}$ ist die Frequenz der elektromagnetische Welle in $\mathrm{Hz}$ und $c$ die Lichtgeschwindigkeit im Medium (Luft) in $\mathrm{m} / \mathrm{s}$ (in Luft ergibt $\operatorname{sich} c=2,99792 \cdot 10^{8} / \overline{n_{\mathrm{a}}} \mathrm{m} / \mathrm{s}$ ).

Wie oben dargelegt, ist die Variation des Brechungsindex selbst bei signifikant großen Schalldruckpegeln nur sehr gering, daher muss bei der Systemauslegung danach getrachtet werden, maximale Rückstreueffizienz zu erzielen. Dazu sind die folgenden Bedingungen wichtig:

a) Bragg-Bedingung:

Die Bragg-Bedingung beschreibt die Abstimmung der elektromagnetischen $\lambda_{\mathrm{e}}$ auf die akustische Wellenlänge $\lambda_{\mathrm{a}}[18]$

$$
\lambda_{\mathrm{e}}=2 \lambda_{\mathrm{a}} .
$$

Ist die elektromagnetische Wellenlänge genau doppelt so groß wie die akustische, dann kommt es $\mathrm{zu}$ einer konstruktiven Interferenz der von den vielen Phasenfronten der akustischen Welle zurückgestreuten Radar-Wellen am Ort des Empfängers [19]. Ausgedrückt in Wellenzahlen lautet die BraggBedingung

$$
k_{\mathrm{a}}=2 k_{\mathrm{e}} .
$$

b) Optimal gewählte Anzahl $N_{\mathrm{B}}$ der Schallwellenzüge. Wird diese klein gewählt, so erhöht sich die Ortsauflösung der Temperaturmessung, da über einer geringere Distanz $\overline{A B}$ gemittelt wird, allerdings sinkt die Rückstreueffizienz sehr stark ab und der Einfluss von

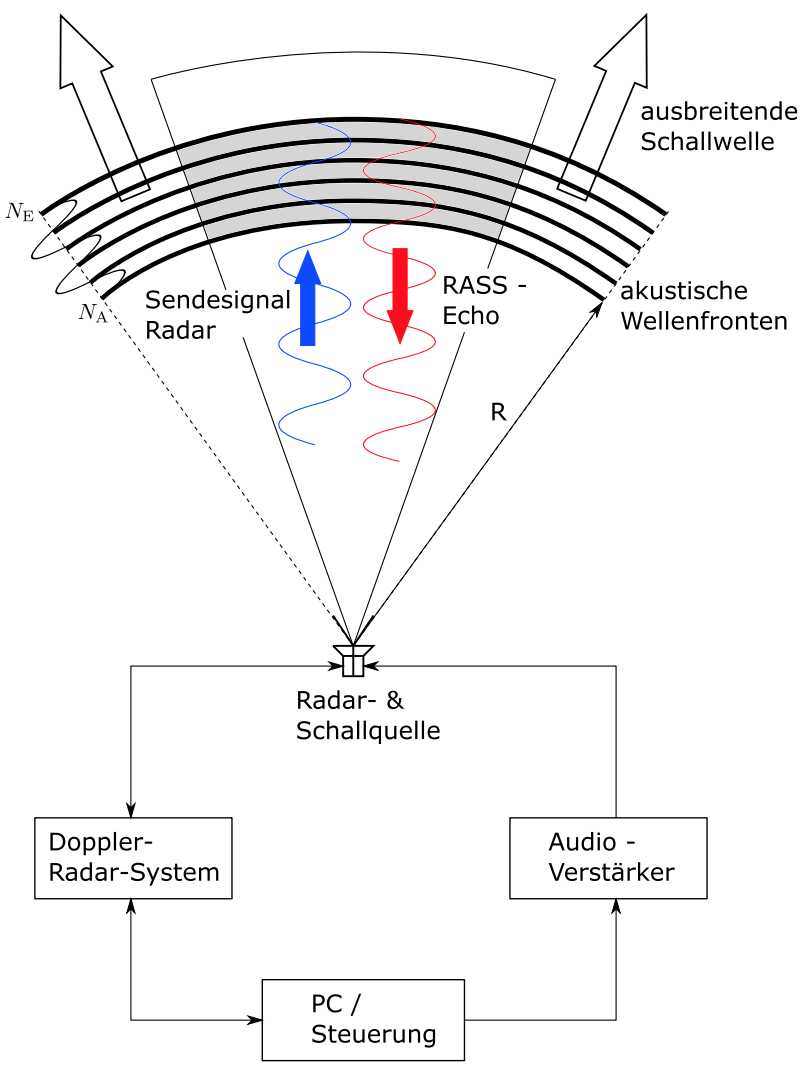

Abb. 1: Bei der Interaktion zwischen der elektromagnetischen mit der akustischen Welle kommt es zu Streuungen des Radar-Signals. Ein geringer Teil, das RASS Echo, wird an die Sende- und Empfangseinheit zurück reflektiert. Das dopplerverschobene, empfangene Signal enthält die Information der lokalen Temperatur [15].

Rauschen bei der Frequenzschätzung nimmt zu. Umgekehrt führt eine Steigerung der Schallwellenzüge zu einer verringerten Ortsauflösung aber einer wesentlichen Verbesserung des Signal zu Rauschverhältnisses im Radar-Empfänger und damit zu einer Frequenzschätzung mit geringerer Varianz.

c) Kollokation:

Die Effizienz des RASS wird verbessert wenn die von den sphärischen Dichtemodulationen rückgestreuten Radar-Wellen sich im Phasenzentrum der Empfangsantenne konstruktiv überlagern. Da die Phasenfronten der elektromagnetischen und auch der akustischen Welle eine jeweils sphärische Form aufweisen, muss erreicht werden, dass deren Phasenzentren zusammenfallen (= konfokale Anordnung der Quellen, Hochtöner und Radar-Antenne). Das bedeutet, dass die Phasenzentren von Hochtöner und Radar-Antenne identisch sein sollten

$$
\vec{n}_{\mathrm{e}}=\vec{n}_{\mathrm{a}}
$$


Tab. 1: Parameter, Beschreibungen und Einheiten für die Gleichung (8).

\begin{tabular}{lll}
\hline Parameter & Beschreibung & Einheit \\
\hline$P_{\mathrm{r}}$ & Empfangsleistung Radar & $\mathrm{W}$ \\
$P_{\mathrm{t}}$ & Sendeleistung Radar & $\mathrm{W}$ \\
$P_{\mathrm{a}}$ & akustische Sendeleistung & $\mathrm{W}$ \\
$A_{\mathrm{a}}$ & Proportionalitätskonstante & $\frac{\mathrm{kg} \mathrm{W}}{\mathrm{m}^{2} \mathrm{~s}}$ \\
$G_{\mathrm{t}}$ & Antennengewinn & $\mathrm{dB}$ \\
$\theta_{\mathrm{r}}$ & Antennenstrahlbreite & $\mathrm{rad}$ \\
$N_{\mathrm{B}}$ & Anzahl der Bragg-Zyklen & 1 \\
$R$ & Abstand Messposition Phasenzentrum & $\mathrm{m}$ \\
$\lambda_{\mathrm{a}}$ & akustische Wellenlänge & $\mathrm{m}$ \\
$\lambda_{\mathrm{e}}$ & elektromagnetische Wellenlänge & $\mathrm{m}$ \\
$k_{\mathrm{a}}$ & akustische Wellenzahl & $\frac{\mathrm{rad}}{\mathrm{m}}$ \\
$k_{\mathrm{e}}$ & elektromagnetische Wellenzahl & $\frac{\mathrm{rad}}{\mathrm{m}}$ \\
\hline
\end{tabular}

$\vec{n}_{\mathrm{a}}$ beschreibt den Wellenvektor (Ausbreitungsvektor) der akustischen Welle und $\vec{n}_{\mathrm{e}}$ stellt den PoyntingVektor der elektromagnetischen Welle dar.

Konkret wird das über ein feines Metallgitter realisiert. Dieses Gitter wirkt für die elektromagnetischen Wellen wie ein Umlenkspiegel und lenkt die RadarWellen in den Ausbreitungspfad der Schallwellen, die sich durch das kleinmaschige Gitter unbeeinflusst ausbreiten [14].

Mit der RASS-Gleichung, welche in [1, 3] genauer betrachtet wurde, kann die vom Radar empfangene Leistung als Funktion von verschiedenen Parametern des Messsystems berechnet werden.

$$
P_{\mathrm{r}}=\frac{A_{\mathrm{a}}^{2} \pi^{2} P_{\mathrm{t}} P_{\mathrm{a}} G_{\mathrm{t}}^{2} \theta_{\mathrm{r}}^{4} N_{\mathrm{B}}^{2}}{16 R^{2}}\left(\frac{\lambda_{\mathrm{a}}}{\lambda_{\mathrm{e}}}\right)^{2} \operatorname{sinc}\left[\left(2 k_{\mathrm{e}}-k_{\mathrm{a}}\right) \frac{N_{\mathrm{B}} \lambda_{\mathrm{a}}}{2}\right]^{2}
$$

In der Tabelle 1 sind alle Parameter mit den entsprechenden Einheiten aufgelistet. Von Gleichung (8) ist zu sehen, dass die Empfangsleistung eine $R^{-2}$ Abhängigkeit aufweist. Das ist auf die sphärisch divergierende akustische Welle zurückzuführen [16, 17]. Weiters ist die Empfangsleistung proportional zum Quadrat der Bragg-Zyklen $N_{\mathrm{B}}^{2}$, zur elektromagnetischen Sendeleistung $P_{\mathrm{t}}$ und zur akustischen Sendeleistung $P_{\mathrm{a}}$. Ein wichtiger Aspekt ist die Maximierung des Funktionswertes der Sinc-Funktion, also der idealerweise vollständig kohärenten Überlagerung der Rückstreuungen. Ersichtlich, wenn die Gleichung (6), also die Bragg-Bedingung, in den Ausdruck für die Empfangsleistung (8) einsetzt wird. Die Kombination von (8) mit dem im nächsten Abschnitt hergeleiteten Signalmodel liefert nun die Grundlage für das optimierte Systemdesign.

\section{Signalverarbeitung}

Die Signalverarbeitungskette gliedert sich grob in folgende Schritte:

a) Nach Aussenden der akustischen Welle, Messdaten des Doppler-Radar aufzeichnen,

b) aus den aufgenommenen Daten die Dopplerfrequenz für verschiedene örtliche Bereiche bestimmen,

c) aus der jeweiligen Dopplerfrequenz die Strömungsgeschwindigkeit bzw. Temperatur berechnen.

Der Messablauf wird in Abschnitt 5 im Detail beleuchtet.

Grundsätzlich handelt es sich bei der Signalverarbeitung der RASS Signale (des Radar-Echos), um das Problem der Schätzung einer Dopplerfrequenz aus sehr schwachen und daher stark verrauschten Empfangssignalen. Daher wird zuerst ein mögliches Signalmodell vorgestellt und anschließend gezeigt, wie aus den aufgenommenen Daten die Dopplerfrequenz optimal geschätzt werden kann.

\section{Signalmodell}

In Abbildung 2 ist eine einfache Realisierung eines monostatischen Doppler-Radars dargestellt. Eine detaillierte Beschreibung der Funktionsweise ist z. B. im bekannten Werk vom Skolnik [20] angeführt, folglich wird nur kurz der Ablauf beschrieben.

Ein Frequenz-Synthesizer (ein in der Frequenz sehr genau regelbarer Oszillator) generiert ein hochfrequentes Mikrowellensignal. Im Sendepfad wird dieses Signal zunächst durch einen Splitter, welcher einen Teil des Signals auch dem im Empfangsteil liegenden Demodulator zur Verfügung stellt, und anschließend durch einen Zirkulator geführt, der eine effektive Isolation des Empfangsteils vom Sendeteil erlaubt [21]. Anschließend wird das Sendesignal über eine Antenne abgestrahlt. Der Zirkulator ist notwendig, da das Radar-System monostatisch ausgeführt ist und somit die Signalrichtungen aufgetrennt werden müssen. Das empfangene, dopplerverschobene RASS-Echo wird über den Zirkulator in den Mischer geleitet. Die einfachste mögliche Modellierung für den Mischvorgang ist eine Multiplikation der beiden Signale [20]. Anschließend wird das nach einer Tiefpass-Filterung verbleibende niederfrequente Signal verstärkt, gefiltert und digitalisiert.

Um die Signalauswertung auf den aktuell interessierenden Ortsbereich $R \leq s<\left(R+\lambda_{\mathrm{a}} \cdot N_{\mathrm{B}}\right)$ einzuschränken, definiert man Indizes, die für diese Grenzen (der floorOperator $\lfloor\ldots\rfloor$ gibt die größte ganze Zahl, die kleiner als 


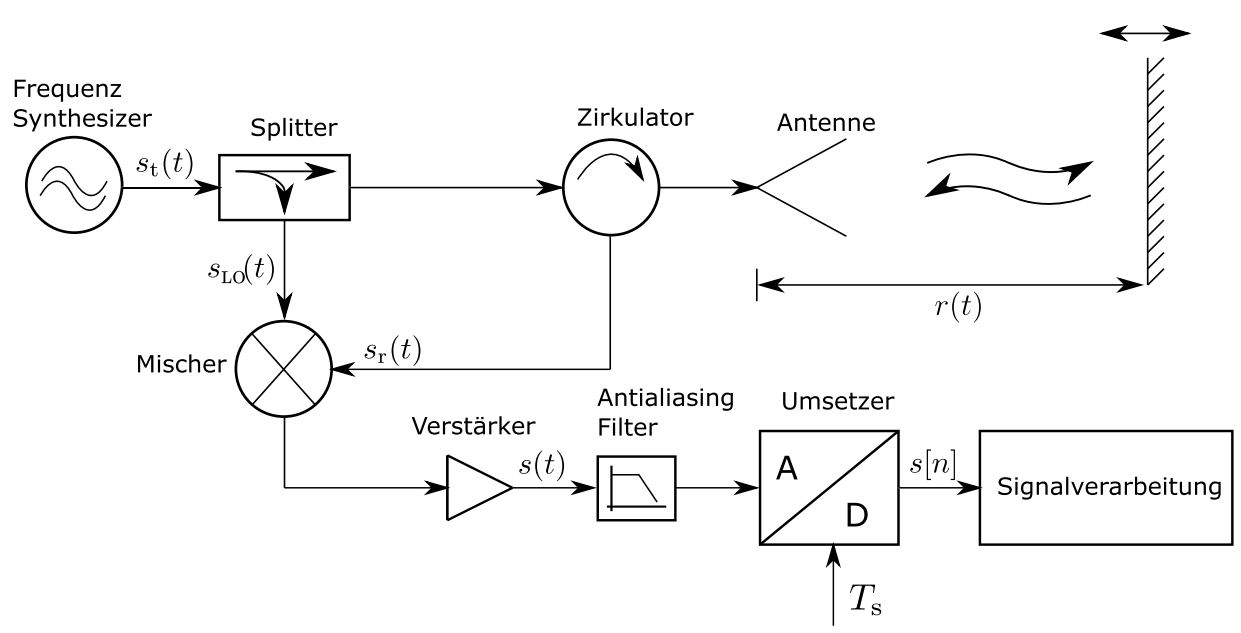

Abb. 2: Mit einem Doppler-Radar wird die Geschwindigkeit eines Schallpulses gemessen. Diese Abbildung zeigt ein Blockschaltbild eines monostatischen kontinuierlichen Doppler-Radars. Für eine detaillierte Beschreibung der Signalentstehung wird auf das bekannte Werk von Skolnik [20] verwiesen.

das Argument ist)

$$
\begin{aligned}
& N_{\mathrm{A}}=\left\lfloor\frac{R}{c_{\mathrm{a}, 0} \cdot T_{\mathrm{s}}}\right\rfloor \\
& N_{\mathrm{E}}=\left\lfloor\frac{R+\lambda_{\mathrm{a}} \cdot N_{\mathrm{B}}}{c_{\mathrm{a}, 0} \cdot T_{\mathrm{s}}}\right\rfloor
\end{aligned}
$$

und ein Analysefenster $\omega(n)$,

$$
\omega(n)=\left\{\begin{array}{lll}
0 & \text { für } & 0 \leq n \leq N_{\mathrm{A}}-1 \\
1 & \text { für } & N_{\mathrm{A}} \leq n \leq N_{\mathrm{E}} \\
0 & \text { für } & N_{\mathrm{E}}+1 \leq n<\infty
\end{array}\right.
$$

das aus dem kontinuierlich digitalisierten Signal nur jene Werte extrahiert, die relativ zum Zeitpunkt der Aussendung des Schallwellenpakets im gesuchten, auszuwertenden Ortsbereich liegen.

Daraus ergibt sich das gefensterte und digitalisierte (Abtastrate $1 / T_{\mathrm{s}}$ ) deterministische Signal $s[n]$, das weiter ausgewertet wird.

$$
\begin{aligned}
s[n] & =A \cdot \cos \left(2 \pi \cdot f_{\mathrm{d}} \cdot n \cdot T_{\mathrm{s}}+\phi\right) \\
& =A \cdot \cos \left[4 \pi \frac{f_{\mathrm{e}}}{c} T_{\mathrm{s}}\left(K_{\mathrm{a}} \cdot \sqrt{\vartheta}+v_{\mathrm{w}}\right) \cdot n+\phi\right]
\end{aligned}
$$

$n$ ist der Abtastindex, mit $n=0,1, \cdots,\left(N_{\mathrm{E}}-N_{\mathrm{A}}\right) . A$ ist die Amplitude der deterministischen Komponente im Signal, $T_{\mathrm{s}}$ das Abtastinkrement. Die zufällige Phase des Signals wird mit $-\pi \leq \phi<\pi$ angenommen. In (9) wurde die Dopplerfrequenz $f_{\mathrm{d}}$ durch den Ausdruck (4) ersetzt. Weiters wurde die Schallgeschwindigkeit-Temperaturbeziehung (1) miteinbezogen.

Da jede reale Messung mit Rauschen kontaminiert ist, wird hier dem abgetasteten Signal nach dem AnalogDigital-Umsetzer (vgl. Abb.2) mittelwertfreies, weißes
Gaußsches Rauschen (AWGR) mit Varianz $\sigma^{2}, w[n] \sim$ $\mathcal{N}\left(0, \sigma^{2}\right)$ überlagert. Das zugehörige Messdatenmodell lautet somit

$$
x(n)= \begin{cases}s[n]+w[n] & \begin{array}{l}
\text { für Schallwellenpaket } \\
\text { im Auswertebereich }
\end{array} \\
=A \cos \left(\xi\left(K_{\mathrm{a}} \sqrt{\vartheta}+v_{\mathrm{w}}\right) n+\phi\right)+w[n] \\
w[n] & \text { für kein auswertbares } \\
& \text { Schallwellenpaket im } \\
& \text { Auswertebereich }\end{cases}
$$

mit $\xi=4 \pi \cdot T_{\mathrm{s}} \cdot f_{e} / c$. In den folgenden Abschnitten stellt diese Gleichung die Grundlage für den Temperaturschätzer, sowie für die Berechnung der Messunsicherheit dar.

Die verwendeten, kommerziellen Komponenten des Messaufbaus sind in der Tabelle 2 aufgelistet. Ausgehend von einem $10 \mathrm{GHz}$ Synthesizer wurde das System so ausgelegt, dass Temperaturen zwischen $-10^{\circ} \mathrm{C}$ und $+50^{\circ} \mathrm{C}$ gemessen werden können. Das bedeutet, dass aufgrund der Bragg-Bedingung (5) Schallfrequenzen zwischen $21,7 \mathrm{kHz}$ und $24,0 \mathrm{kHz}$ erforderlich sind. $\mathrm{Zu}$ diesem Zweck wurde der Hochtöner Ground Zero GZCT 0500X [22] gewählt. Dieser erlaubt einen Schalldruckpegel von zumindest $90 \mathrm{~dB}$ (ref. $2 \cdot 10^{-5} \mathrm{~Pa}$ ) zu erzeugen.

\section{Schätzer für die Dopplerfrequenz}

Wie aus (9) leicht erkennbar ist, ist die gesuchte Temperatur- bzw. Strömungsgeschwindigkeit in der Frequenz des auf das Basisband gemischten Radar-Signals enthalten. Somit kann die RASS Messung grundlegend 
Tab. 2: Verwendete Komponenten für den realen Systemaufbau. Alle verwendeten Bauteile sind kommerziell erhältlich.

\begin{tabular}{ll}
\hline Radar-System & \\
\hline CW Sendefrequenz & 10 GHz \\
\hline HF - Synthesizer & NI QuickSyn Lite FSL-0020 \\
HF - Splitter & Mini-Circuits ZX10-2-183+ \\
HF - Verstärker & Mini-Circuits ZX60-24+ \\
HF - Zirkulator & Pasternack PE8403 \\
HF - Mischer & Mini-Circuits ZX05-24MH+ \\
Antenne & Flann Gain Horn Series 16240-20 \\
Variabler Verstärker & Analog Devices AD8331-EVAL \\
ADC & NI 6356 \\
\hline Akustisches Setup & \\
\hline DAC & NI 6356 \\
Verstärker & Peavey CS4000 \\
Hochtöner 1 & Ground Zero GZCT 0500X \\
\hline
\end{tabular}

auf ein Frequenzschätzproblem zurückgeführt und auch dementsprechend analysiert werden.

Für den Fall von additivem, Gaußschem Rauschen kann allgemein gezeigt werden, dass die diskrete FourierTransformation (DFT) ein erwartungstreuer Schätzer mit minimaler Varianz ist. Da für das angegebene Signalmodell entweder eine einzelne starke Spektralkomponente oder nur ein Rauschspektrum zu erwarten ist, ergibt sich die Frequenzschätzung aus der Position eines möglichen Maximums des Betragsspektrums [24]

$$
\widehat{f_{\mathrm{d}}}=\arg \max _{f}\left\{\left|\sum_{n=0}^{N_{\mathrm{A}}-N_{\mathrm{E}}} x[n] \cdot \mathrm{e}^{-\mathrm{j} 2 \pi f T_{\mathrm{s}} n}\right|^{2}\right\} .
$$

Die Frequenz der maximalen Spektralkomponente stellt die geschätzte Dopplerfrequenz dar. Das Zirkumflex über der Dopplerfrequenz $\widehat{f_{\mathrm{d}}}$ weist auf einen Schätzwert hin. Aus der Kombination von (1) und (10) folgt für die zu schätzende Temperatur $\widehat{\vartheta}$

$$
\widehat{\vartheta}=\left(\frac{\widehat{f_{\mathrm{d}}} \cdot c}{2 \cdot f_{\mathrm{e}} \cdot K_{\mathrm{a}}}\right)^{2} .
$$

\section{Unsicherheit der Frequenzschätzung}

Bei industriellen Anwendungen ist es notwendig, die $\mathrm{zu}$ erwartende Messunsicherheit eines Messsystems zu kennen. Mit Hilfe der Cramér-Rao-Schranke (CR-Schranke) kann eine untere Grenze für die erreichbare Schätzvarianz [24]

$$
\operatorname{var}\left\{\widehat{\beta}_{i}\right\} \geq\left[\mathbf{F}^{-1}(\beta)\right]_{i i}
$$

der zu schätzenden Parameter $\beta=\left[\vartheta v_{\mathrm{w}}\right]^{T}$ für einen erwartungstreuen Schätzer berechnet werden.

Der Übersichtlichkeit halber wird zunächst die unbekannte Amplitude und Phase ignoriert. $\mathbf{F}$ beschreibt eine $2 \times 2$ große Fisher Informationsmatrix (FIM). Für ein additives, weißes, Gaußsches Messrauschen, wie es in [24] diskutiert wird, kann die FIM geschlossen berechnet werden mit

$$
[\mathbf{F}(\boldsymbol{\beta})]_{i j}=\frac{1}{\sigma^{2}} \sum_{n=0}^{N-1} \frac{\partial s[n ; \boldsymbol{\beta}]}{\partial \beta_{i}} \frac{\partial s[n ; \boldsymbol{\beta}]}{\partial \beta_{j}} .
$$

Für das konkrete Signalmodell (9) berechnet sich die FIM $\mathrm{zu}$

$$
\mathbf{F}(\boldsymbol{\beta}) \approx \frac{\xi^{2} A^{2} N^{3}}{\sigma^{2} 6}\left[\begin{array}{rr}
\frac{K_{\mathrm{a}}^{2}}{49} & \frac{K_{\mathrm{a}}}{2 \sqrt{9}} \\
\frac{K_{\mathrm{a}}}{2 \sqrt{9}} & 1
\end{array}\right] .
$$

Für die Berechnung der Varianzen (12) muss die Inverse der FIM berechnet werden. Bei der Überprüfung für die Existenz der Inversen von (14) zeigt sich, $\operatorname{dass} \operatorname{det}(\mathbf{F})=0$ gilt, es sich also um eine singuläre Matrix handelt und somit kein erwartungstreuer Schätzer mit endlicher Varianz existiert [26]. Die Temperatur $\vartheta$ und die Strömungsgeschwindigkeit $v_{\mathrm{w}}$ können daher nicht unabhängig voneinander geschätzt werden. Ist jedoch einer dieser Parameter bekannt, so ist es möglich den jeweils anderen Parameter zu schätzen, bzw. die CR-Schranke zu berechnen. Werden die messtechnisch irrelevanten, unbekannten Parameter Amplitude und Phase miteinbezogen, so ändert sich die Situation nicht, nur die Berechnung der CR-Schranke gestaltet sich umfangreicher.

Unter der notwendigen Annahme der bekannten Temperatur erhält man nach kurzer Herleitung eine untere Schranke für die Schätzvarianz zur Strömungsgeschwindigkeit (mit $\left.N=N_{\mathrm{E}}-N_{\mathrm{A}}+1\right)$

$$
\operatorname{var}\left\{\widehat{v_{\mathrm{w}}}\right\} \geq \frac{c^{2}}{4 f_{\mathrm{e}}^{2}} \frac{12}{(2 \pi)^{2} \eta T_{\mathrm{s}}^{2} N^{3}} .
$$

Der Parameter $\eta$ beschreibt dabei den Signal-Rauschabstand (SRA) [24, 25]

$$
\eta=A^{2} /\left(2 \sigma^{2}\right)
$$

Ähnliches gilt auch bei bekannter Strömungsgeschwindigkeit. Eine untere Schranke für die Varianz der Temperaturschätzung lautet dann

$$
\operatorname{var}\{\hat{\vartheta}\} \geq \frac{c^{2} \vartheta^{2}}{K_{\mathrm{a}}^{2} f_{\mathrm{e}}^{2}} \frac{12}{(2 \pi)^{2} \eta T_{\mathrm{s}}^{2} N^{3}} .
$$


Wendet man den Ausdruck $T_{\mathrm{s}}^{2} N^{2}=\left(\lambda_{\mathrm{s}}^{2} N_{\mathrm{B}}^{2}\right) / c_{\mathrm{a}}^{2}$ auf (17) an, so vereinfacht sich der Ausdruck für die Varianz der Temperaturschätzung zu

$$
\operatorname{var}\{\widehat{\vartheta}\} \geq \frac{12 \vartheta^{2}}{\pi^{2} \eta N N_{\mathrm{B}}^{2}}
$$

Aus (18) kann geschlossen werden, dass sich die Varianz der Temperaturschätzung proportional zum Quadrat aktuellen Temperatur $\vartheta$ bzw. indirekt proportional zum Quadrat der Anzahl der Bragg-Zyklen $N_{\mathrm{B}}^{2}$ ändert.

Aus (8) erkennt man, dass die akustische Sendeleistung $P_{\mathrm{a}}$ die elektromagnetische Empfangsleistung beeinflusst und somit auch entsprechend den SRA. Teilt man den SRA gezielt in einen Faktor $\eta_{0}$ und in einen Faktor $P_{\mathrm{a}} N_{\mathrm{B}}^{2}$ auf, so lässt sich die akustische Sendeleistung explizit im Ausdruck für die Varianz der Temperaturschätzung (18) angeben zu

$$
\operatorname{var}\{\widehat{\vartheta}\} \geq \frac{12 \vartheta^{2}}{\pi^{2} \eta_{0} P_{\mathrm{a}} N N_{\mathrm{B}}^{4}} .
$$

Aus Gleichung (19) sieht man, dass die Varianz der Temperaturschätzung stark (4. Potenz) von der Anzahl der Bragg-Zyklen $N_{\mathrm{B}}$ abhängt. Diese Überlegungen können für einen optimalen Systementwurf in Betracht gezogen werden.

Referenzmessungen mit einem Thermoelement bestätigen die Erwartungstreue des Schätzers, soweit dies die Grundgenauigkeit des Thermoelements zulässt (vgl. auch Abschnitt 5). Zur Verifikation der CR-Schranke wurden Temperaturmessungen (500 Messreihen) durchgeführt und daraus die Standardabweichung berechnet. Die vorhergesagten Varianzen werden dabei ebenfalls auf eine Standardabweichung umgerechnet. Eine genaue Beschreibung des Messablaufes wird im nächsten Abschnitt gegeben. Die Messungen wurden in einem Labor bei einer Temperatur von $\approx 23^{\circ} \mathrm{C}$ durchgeführt und bei einem Messabstand von $R=5 \mathrm{~m}$ ausgewertet. Abbildung 3 zeigt für verschiedene Schallpulslängen die ermittelte Standardabweichung der Temperaturschätzung im Vergleich zu den vorhergesagten Werten, welche mit (19) berechnet werden können. Es ist gut zu erkennen, dass mit größer werdender Schallpulslänge, also mehr Bragg-Zyklen, die Unsicherheit bei der Temperaturmessung verringert wird. Bei einem SRA von $-9,07 \mathrm{~dB}$ beträgt die vorhergesagte Standardabweichung $0,21^{\circ} \mathrm{C}\left(-6,69 \mathrm{~dB}\right.$ bei $1{ }^{\circ} \mathrm{C}$ Referenzwert) und die aus den gemessenen Temperaturen berechnete Standardabweichung $0,24^{\circ} \mathrm{C}(-6,19 \mathrm{~dB})$. Das bedeutet eine Abweichung von $0,03^{\circ} \mathrm{C}$.

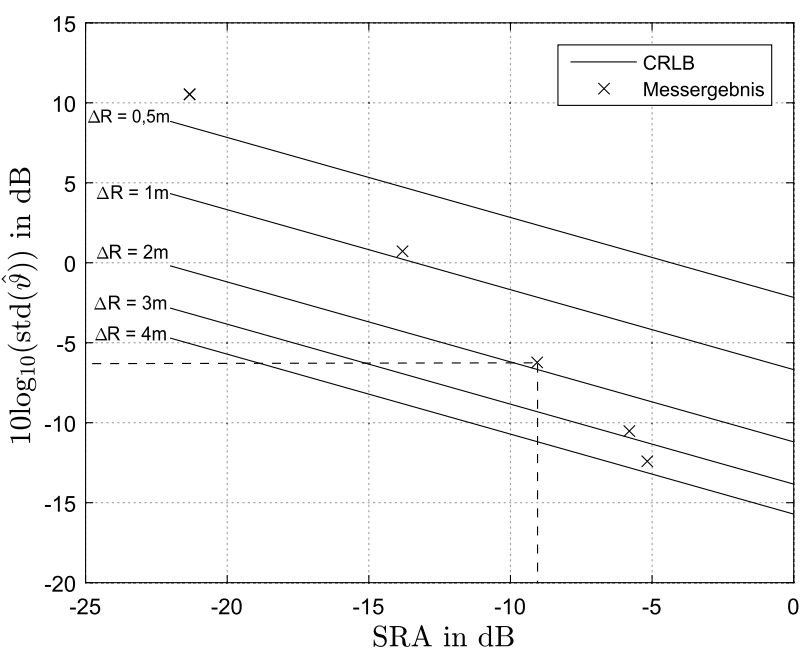

Abb. 3: Für verschiedene Schallwellenpaketlängen wurde die Unsicherheit berechnet und mit realen Messdaten verifiziert. Bei einem SRA von $-9,07 \mathrm{~dB}$ und bei einer Schallpulslänge von $2 \mathrm{~m}$ (entspricht 133 Bragg-Zyklen) kommt es zu einer Abweichung von $0,03^{\circ} \mathrm{C}$. Zusätzlich wurde der Effekt der Amplitudenmodulation, verursacht z. B. durch das nichtideale Verhalten des Lautsprechers und Fensterung bei der DFT berücksichtigt.

\section{Optimale Systemauslegung}

Wie bereits erwähnt können die Beziehungen für die Fehlergrenzen für eine optimale Systemauslegung herangezogen werden. Somit kann bereits vor dem konkreten Systemaufbau abgeklärt werden, ob mit den ausgewählten Komponenten die Messanforderungen hinsichtlich geforderter Messunsicherheit erreicht werden kann. Da eine der zu erwartenden Temperatur entsprechende Schallwellenlänge emittiert werden muss und Hochtöner einen stark schwankenden Frequenzgang aufweisen, muss dieser mit einbezogen werden.

In Abbildung 4 ist die zu erwartende Standardabweichung der Temperaturschätzung unter Einbeziehung des Frequenzganges des Hochtöners Ground Zero GZCT 0500X [22] dargestellt. Die Berechnung wurde für einen $2 \mathrm{~m}$ langen Schallpuls durchgeführt. Eine höhere Schallfrequenz $f_{\mathrm{a}}$ bedeutet eine kleinere Schallwellenlänge $\lambda_{\mathrm{a}}$ und somit mehr auswertbare Bragg-Zyklen in diesem $2 \mathrm{~m}$ langen Schallpuls. Allerdings ist in der Abbildung 4 ersichtlich, dass die Standardabweichung der Temperaturschätzung mit zunehmender Schallfrequenz nicht kontinuierlich abnimmt. Das ist auf den Frequenzgang des Hochtöners zurückzuführen, da dieser lokale Maxima des Schalldruckpegels aufweist. Bei etwa $13,5 \mathrm{kHz}$ beträgt die Standardabweichung $0,19^{\circ} \mathrm{C}$. Messungen bei dieser im Hörbereich liegenden Frequenz wurden bewusst vermieden. 


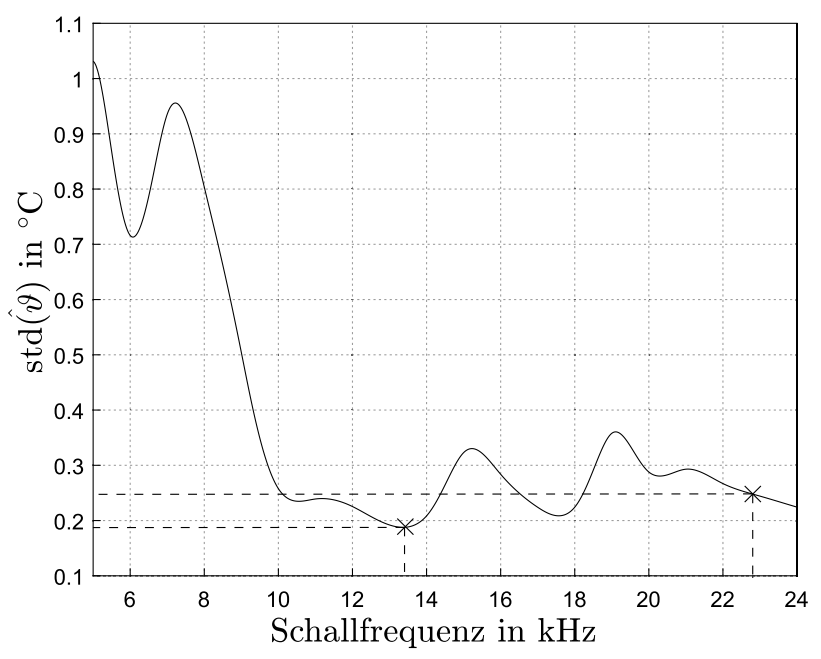

Abb. 4: Diese Abbildung zeigt die Standardabweichung der Temperaturschätzung wenn der Frequenzgang des Hochtonlautsprechers Ground Zero GZCT 0500X [22] in die Berechnung der CR-Schranke mit einbezogen wird. Bei etwa $13,5 \mathrm{kHz}$ beträgt die Standardabweichung $0,19^{\circ} \mathrm{C}$. Diese Darstellung kann nun als Werkzeug zum Systementwurf direkt herangezogen werden. Das heißt, die optimale Sendefrequenz kann direkt abgelesen werden.

\section{Messergebnis}

\section{Messablauf}

Eine schematische Darstellung des Messablaufes ist in Abbildung 5 dargestellt. Zur Verbesserung des SRA werden die Signale von $M$ mit einem typischen $M=200$ Messungen zunächst kohärent gemittelt und dann ausgewertet. $\mathrm{Zu}$ diesem Zweck müssen die Schallpulse immer zu vordefinierten Zeitpunkten abgestrahlt werden. Dieser Vorgang wird über einen Trigger gestartet, der immer nach der Zeitspanne $T_{\mathrm{Pa}}$ gesetzt wird. Zeitgleich zum Aussenden der Schallpulse wird mit dem Doppler-Radar kontinuierlich gemessen, das Basisbandsignal analog-digital umgesetzt und in einem Datenvektor abgespeichert. $\mathrm{Zu}$ jedem Zeitpunkt $T_{\mathrm{Pa}}$ wird eine Messsequenz ausgelöst. Je nach notwendigem SRA und maximal tolerierbarer Messdauer werden $M$ Messungen kohärent gemittelt (vgl. Abb.5, gemittelte Daten). Dieser Datensatz enthält die Information der Ausbreitungsgeschwindigkeit des Schallpulses entlang des Messabstandes. Je nachdem, an welchem Ort $s$ mit $R \leq s<R+\lambda_{\mathrm{a}} N_{\mathrm{B}}$ man die Geschwindigkeit des Schallpulses bestimmen möchte, muss ein entsprechendes Datenfenster betrachtet werden (siehe Abb. 5, Datenfenster). Die selbst wieder vom Verlauf der örtlichen Schallgeschwindigkeit zwischen Phasenzentrum und gewünschtem Messort abhängige Position des Schallpulses wird durch Aufintegration der schallgeschwindigkeitsgewichteten Lauf-

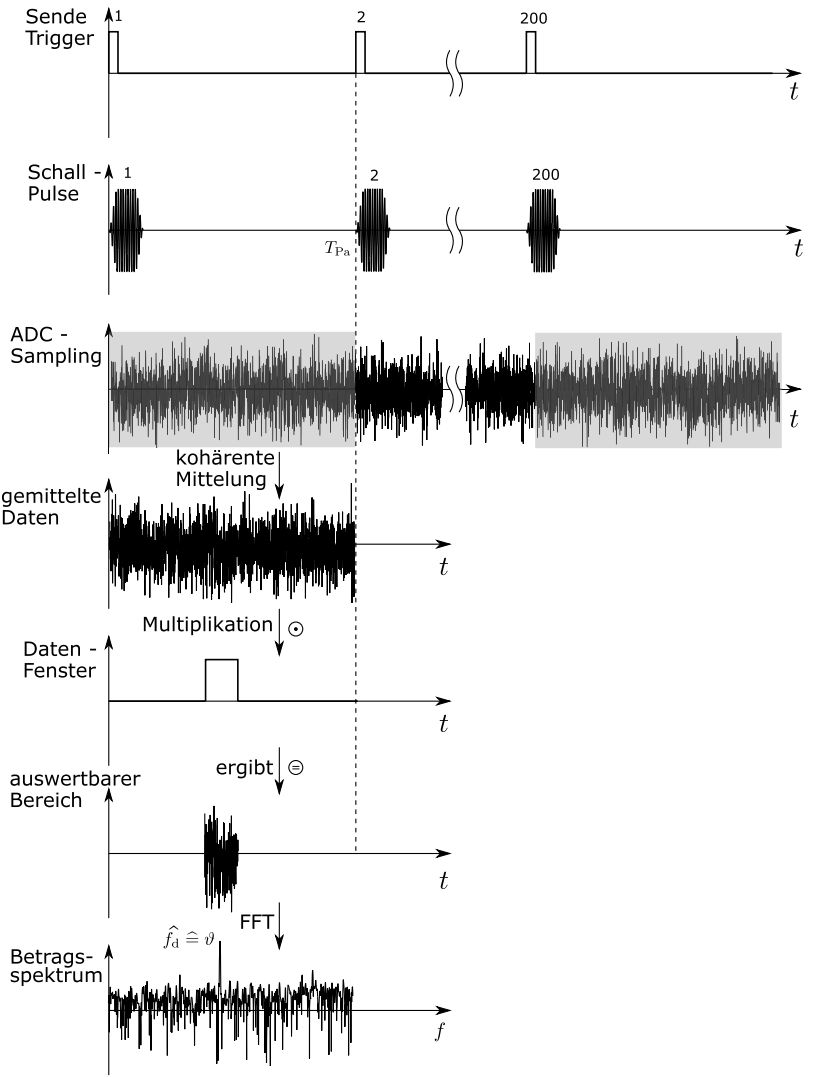

Abb. 5: Schematische Darstellung des Messablaufes. Synchron zum Aussenden der Schallpulse wird mit einem Doppler-Radar kontinuierlich gemessen. Aus dem gemittelten Radar-Signal wird eine Spektralanalyse durchgeführt um die Information der Temperatur zu erhalten.

zeiten bestimmt. Für Details sei auf [9] verwiesen. Mittels der Fast-Fourier Transformation wird aus dem Signal des betrachteten Datenfensters eine Spektralanalyse durchgeführt. Die dadurch bestimmte Dopplerfrequenz ist proportional zur lokalen Ausbreitungsgeschwindigkeit des Schallpulses und somit zur lokalen Temperatur des Gases.

\section{Messung eines Temperaturprofiles}

Nur bei einer optimal eingehaltenen Bragg-Bedingung kommt es zu einer effizienten Rückstreuung und somit zu einem bestmöglich detektierbaren Signal. Da die Schallgeschwindigkeit von der Temperatur abhängig ist (1) kann nur ein eingeschränkter Temperaturbereich von einer Frequenzkombination (Radar und Schall) abgedeckt werden. Für die Messung eines Temperaturprofiles müssen an verschiedene Temperaturen angepasste Schallfrequenzen hintereinander ausgesendet werden. Bei diesem sogenannten Mehrfrequenzverfahren muss der Abstand zwi- 


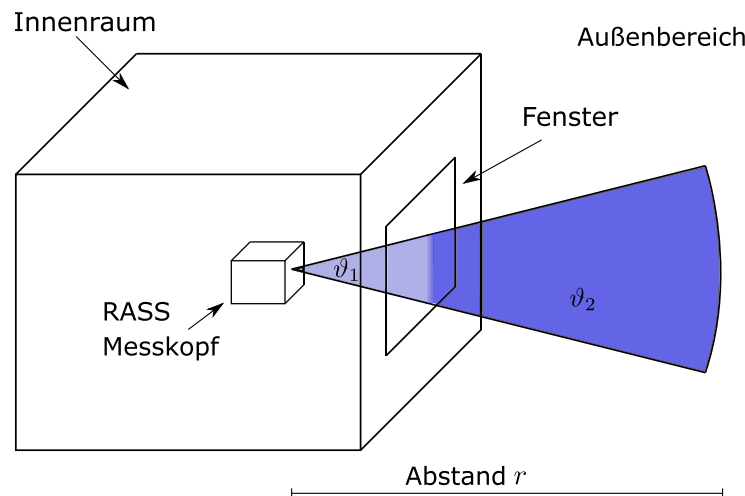

Abb. 6: Zur Messung eines Temperaturprofils wurde das Messsystem in einem Innenraum platziert und darauf geachtet, dass die Messachse bei offenem Fenster nach außen weist. $\vartheta_{1}$ beschreibt die Innenraumtemperatur und $\vartheta_{2}$ die Temperatur im Außenbereich.

schen den Frequenzen so gewählt werden, dass keine Lücken im Temperaturmessbereich entstehen, aber trotzdem die Effizienz der Rückstreuung möglichst hoch bleibt.

Zur Messung eines Temperaturprofiles wurde das System mittig in einem Laborraum positioniert und der Messkopf so ausgerichtet, dass die Messachse bei offenem Fenster nach außen weist. Eine genaue Beschreibung des Messkopfes wurde von den Autoren in [14] vorgestellt. In der Abbildung 6 ist beispielhaft der Messaufbau mit den unterschiedlichen Temperaturen $\vartheta_{1}$ und $\vartheta_{2}$ dargestellt. Zum Zeitpunkt der Messung (27. März 2018) betrug die Außentemperatur $\vartheta_{2}=5^{\circ} \mathrm{C}$ und die Innenraumtemperatur $\vartheta_{1}=27^{\circ} \mathrm{C}$, gemessen mit einem Pt-100 Temperaturfühler (Grenzabweichung $\pm 0,1^{\circ} \mathrm{C}$ ). Damit es zu keinem vorzeitigen Temperaturaustausch kommt, wurde das Fenster erst kurz vor der Messung geöffnet. Für $10^{\circ} \mathrm{C}$ und $24^{\circ} \mathrm{C}$ wurden angepasste Schallfrequenzen $\left(f_{1}=22,502 \mathrm{kHz}, f_{2}=23,052 \mathrm{kHz}\right)$ ausgesendet. Wie in [3] zu sehen, kann damit ein Temperaturbereich von jeweils $\pm 8^{\circ} \mathrm{C}$ abgedeckt und trotzdem noch das Signal detektiert werden.

Für diese Messung wurden $N_{\mathrm{B}}=33$ Bragg-Zyklen gewählt, somit beträgt die Schallpulslänge $0,5 \mathrm{~m}$. Abbildung 7 zeigt das ausgewertete Temperaturprofil, wobei für $R$ alle $20 \mathrm{~cm}$ Temperaturwerte ausgewertet wurden. Auf der Abszisse ist der Abstand zwischen dem Messkopf und dem Schallpulsanfang dargestellt. Das heißt bei einem Abstand von z. B. 0,5 m befindet sich der Schallpuls zwischen $0,5 \mathrm{~m}$ und $1 \mathrm{~m}$ von den Quellen entfernt. Damit ein aussagekräftiges Ergebnis erzielt werden kann, wurden $M=$ 200 Messungen gemittelt. Die gesamte Messzeit betrug $4 \mathrm{~s}$. Somit konnte gewährleistet werden, dass der Wärmeaustausch nur im geringen Maße stattfand. Es ist gut zu erkennen, dass der Messkopf etwa 2,3 $\mathrm{m}$ vor dem Fenster positio-

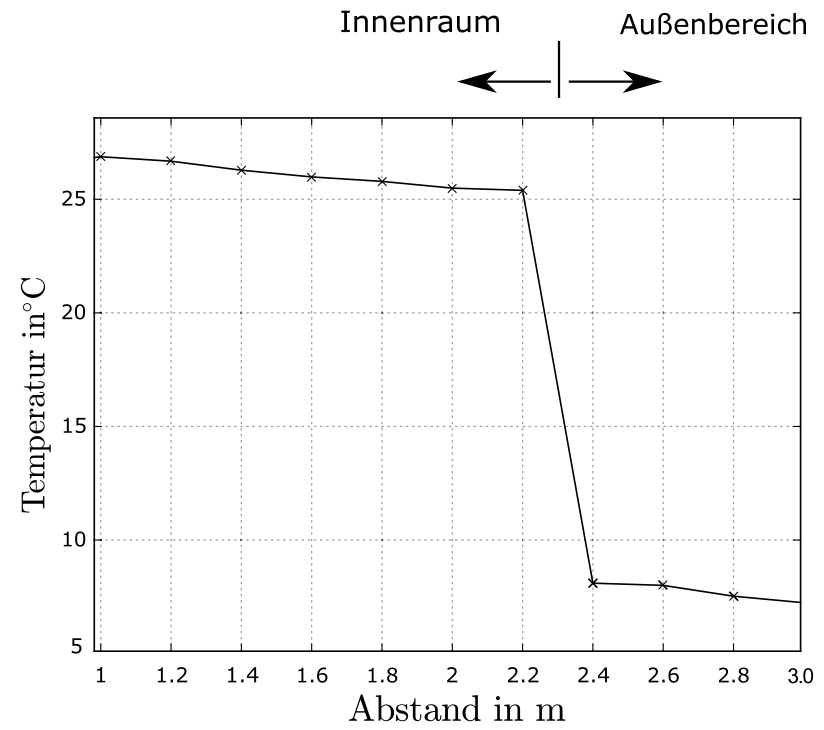

Abb. 7: Das RASS wurde etwa 2,3 m vor dem Fenster positioniert. Obwohl das Fenster erst kurz vor der Messung geöffnet wurde und die Messzeit nur $4 \mathrm{~s}$ betrug ist bereits ein Temperaturdrift entlang der Messachse erkennbar [14].

niert war. Trotz geringer Messzeit kam es bereits zu einem Temperaturdrift entlang des Messpfads.

\section{Zusammenfassung}

Mit dem RASS können berührungslos Temperaturen und Strömungsgeschwindigkeiten von Gasen gemessen werden. Bei industriellen Anwendungen ist es wichtig die Messunsicherheit eines Systems zu kennen. $\mathrm{Zu}$ diesem Zweck wurde in diesem Artikel die CR-Schranke berechnet, die eine Grenze für die erreichbare Schätzgenauigkeit angibt. Weiters wurde gezeigt, dass mit einem Mehrfrequenzverfahren auch Temperaturprofile gemessen werden können.

Da die Temperatur und die Strömungsgeschwindigkeit in Ausbreitungsrichtung der elektromagnetischen Welle nicht unabhängig voneinander geschätzt werden können, ist eine gleichzeitige Bestimmung dieser Parameter mit nur einem System nicht möglich und stellt eine Limitierung dar. Abhilfe würde eine entsprechende Anordnung von mehreren Systemen schaffen.

Danksagung: Besonderer Dank für viele wissenschaftliche und fachliche Diskussionen geht an Frau Vera Ganglberger sowie die Herren Stefan Schuster, Stefan Scheiblhofer, Dominik Zankl und Johann Reisinger ebenfalls von der voestalpine Stahl GmbH. 
Finanzierung: Die Autoren bedanken sich ausdrücklich für die finanzielle Unterstützung durch die Österreichischen Forschungsförderungsgesellschaft (FFG) unter der Projektnummer 861570 sowie bei der voestalpine Stahl $\mathrm{GmbH}$.

\section{Literatur}

1. Stuart Bradley, Atmospheric Acoustic Remote Sensing, CRC Press, 2008.

2. M. Weiß, R. Knöchel, Monostatic Radio-Acoustic Sounding System, In: Geoscience and Remote Sensing Symposium, Hamburg, Germany, S. 29-31, 2001.

3. D. Exel, S. Schuster, B. Zagar, S. Scheiblhofer, D. Zankl, V. Ganglberger, J. Reisinger, Key Design Elements of a Radio-Acoustic-Sounding System for Industrial Applications, In: Sensoren und Messsysteme, Deutschland, Nürnberg, 2019.

4. R. Lerch, G. Sessler, D. Wolf, Technische Akustik: Grundlagen und Anwendungen, Springer, 2009.

5. F. Bernhard, Handbuch der Technischen Temperaturmessung, 2nd ed. Springer, 2014.

6. C. Feilmayr, S. Schuster, G. Lengauer, L. Stegfellner, B. Lackner, E. Schuster, D. Zankl, A. Stelzer, R. Feger, R. Winkel, Burden Surface Scanning via Massive MIMO Imaging Radar in an Ironmaking Blast Furnace, In: METEC, 2015.

7. C. Feilmayr, S. Schuster, V. Ganglberger, B. Lackner, D. Zankl, L. Stegfellner, Innovative Tools for Process Optimization - Burden Surface Scanning via Beamforming Radar and Measurement of the Top Gas Temperature Distribution, In: 7th European Coke and Ironmaking Congress, ECIC, 2016.

8. J.A. Saffold, F. Williamson, K. Ahuja, L. Stein, M. Muller, Radar-acoustic Interaction for IFF Applications, In: Radar Conference, Waltham USA, S. 198-202, 1999.

9. D. Exel, S. Schuster, K. Thaller, B. Zagar, D. Zankl, S. Scheiblhofer, V. Ganglberger, J. Reisinger, Radio-Acoustic-Sounding System - berührungslose u. abstandsaufgelöste akustische Temperaturmessung im industriellen Umfeld, In: Elektrotechnik und Informationstechnik, S. 389-395, 2018.

10. J. M. Marshall, A. M. Peterson, A. A. Barnes, Combined radar-acoustic sounding system, Applied Optics, Vol.11, No.1, 108-112, 1972.

11. S. F. Clifford, T. I. Wang, The range limitation on radar-acoustic sounding system (RASS) due to atmospheric refractive turbulence, IEEE Transactions on Antennas and Propagation, Vol. 25, 319-326, 1977.

12. A. M. Angevine, Radio Acoustic Sounding System (RASS) Application and Limitations, In: Geoscience and Remote Sensing Symposium, Honolulu, USA, S. 1180-1182, 2000.

13. J. R. Kutia, W. Xu, A Portable Radio-Acoustic Sounding System for Temperature Profiling Within the Boundary Layer, In: Industrial Electronics Society, IECON, S. 2308-2313, 2014.

14. D. Exel, B. Zagar, S. Schuster, S. Scheiblhofer, D. Zankl, V. Ganglberger, J. Reisinger, Ein kompaktes Radio-Acoustic -Sounding System zur Temperatur und Strömungsgeschwindigkeitsmessung, In: 6. Tagung Innovation Messtechnik, Österreich, Linz, 2019.
15. B. B. Balsley, K. S. Gage, The MST radar technique: Potential for middle atmospheric studies, Pure and Applied Geophysics, Vol. 118, 452-493, 1980.

16. M. Daas, R. Knöchel, Compact Sounding System using Microwaves and Ultrasound, In: Microwave Symposium Digest, Long Beach, USA, S. 257-260, 1989.

17. M. Daas, R. Knöchel, Microwave-Acoustic Measurement System for Remote Temperature Profiling in Closed Envirenments, In: Microwave Conference, Helsinki, Finland, S. 1225-1230, 1992.

18. M. Weiß, R. Knöchel, A Monostatic Radio-Acoustic Sounding System Used as an Indoor Remote Temperature Profiler, IEEE Transaction on Instrumentation and Measurement, Vol.50, No. 5, 2001.

19. Benjamin Moussa Daas, Die elektromagnetisch-akustische Wechselwirkung und ihre Anwendung zur berührungslosen Entfernungs- und Temperaturmessung in geschlossenen Räumen, Shaker Verlag, Aachen, 1996. ISBN 3-8265-2148-X.

20. M. I. Skolnik, Introduction to Radar Systems, McGraw-Hill Education, 2003.

21. F. Gustrau, Hochfrequenztechnik, Grundlagen der mobilen Kommunikation, Hanser Verlag, 2013.

22. Ground Zero GZCT-0500X, Datenblatt: https://www.groundzero-audio.com/de/produkt/gzct-0500x/.

23. E. Schrüfer, L. Reindl, B. Zagar, Elektrische Messtechnik, Hanser Verlag, 2018.

24. S. M. Kay, Fundamentals of Statistical Signal Processing Estimation Theory, Prentice Hall, Englewood Cliffs, Upper Saddle River, NJ, 1993.

25. S. Schuster, D. Exel, B. Zagar, S. Scheiblhofer, D. Zankl, V. Ganglberger, J. Reisinger, Noise Variance and Signal-to-Noise Ratio Estimation from Spectral Data, In: IEEE International Instrumentation and Measurement Technology, Neuseeland, Auckland, 2019.

26. P. Stoica and T. L. Marzetta, Parameter estimation problems with singular information matrices, IEEE Trans. Signal Processing, Vol. 49, No.1, pp. 87-90, Jan 2001.

\section{Autoreninformationen}

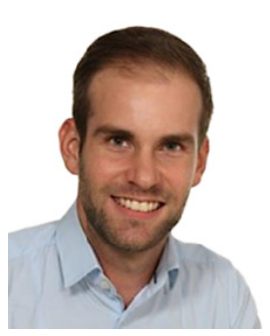

\author{
Dominik Exel \\ Johannes Kepler Universität Linz, Institut \\ für Elektrische Messtechnik, \\ Altenbergerstraße 69, 4040 Linz, Austria \\ dominik.exel@jku.at
}

Dr. Dominik Exel, M. Sc. studierte Automatisierungstechnik an der Fachhochschule Wels und war von 2016-2020 am Institut für Elektrische Messtechnik an der Johannes Kepler Universität in Linz beschäftigt. Im Rahmen seiner Dissertation beschäftigte er sich mit der berührungslosen Temperaturmessung. Seine Interessen liegen im Bereich der Signalverarbeitung und Sensorsysteme. 


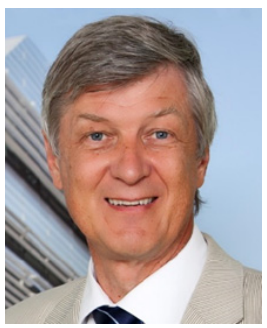

\section{Bernhard Zagar}

Johannes Kepler Universität Linz, Institut für Elektrische Messtechnik, Altenbergerstraße 69, 4040 Linz, Austria bernhard.zagar@jku.at

Univ.-Prof. Dipl.-Ing. Dr. Bernhard G. Zagar leitet das Institut für Elektrische Messtechnik an der Johannes Kepler Universität Linz. Seine Interessen sind im Fachgebiet Messtechnik weit gestreut und erstrecken sich vom Sensordesign bis hin zur Signalverarbeitung. Besondere Schwerpunkte liegen im Bereich der optischen Messtechnik, der Bildverarbeitung und der Magnettomographie. 